\title{
A model for molecular adsorption on the surface of a columnar structure including steric effects and adsorbate-adsorbate repulsive interactions
}

\author{
M.M. Gómez a, J.M. Vara ${ }^{\text {a,1 }}$, J.C. Hernández ${ }^{\text {a }}$, R.C. Salvarezza ${ }^{\text {b }}$, A.J. Arvia ${ }^{\text {b,* }}$ \\ ${ }^{a}$ Departamento de Química-Física Aplicada, Facultad de Ciencias, Universidad Autónoma, Madrid, Spain \\ ${ }^{\mathrm{b}}$ Instituto de Investigaciones Fisicoquímicas Teóricas y Aplicadas (INIFTA) (Universidad de La Plata-CONICET), Sucursal 4, \\ Casilla de Correo 16, 1900 La Plata, Argentina
}

Received 31 January 2001; received in revised form 4 July 2001

\begin{abstract}
Two-dimensional Monte Carlo simulations for adsorption on a columnar structured substrate were made to explore the steric effects of pore width and depth on molecular adsorption under adsorbate-adsorbate repulsive interactions. Simulation adsorption data fit a Frumkin-type isotherm for each set of pore width, depth and top column dimensions. As pore depth increases and pore width approaches the adsorbate molecular size, the surface coverage by adsorbed particles decreases sharply. Therefore, the adsorbate-adsorbate interaction parameters derived from the simulated isotherms exhibit a marked dependence on the substrate roughness factor $(R)$. Phenomenologically, the influence of $R$ on the isotherms is smoothed down by introducing a correction factor $(f)$, which allowed us to collapse all simulated isotherms into a single isotherm. Results from the model are qualitatively compared to earlier observations about the influence of columnar structured gold surface roughness on the adsorption of 1,10-phenantroline in acid solution (oph) at constant potential $\left(E>E_{\mathrm{pzc}}\right)$. (C) 2001 Elsevier Science Ltd. All rights reserved.
\end{abstract}

Keywords: Adsorption at columnar surfaces; Adsorption modelling; Monte Carlo simulation; 1,10-Phenantroline adsorption on gold

\section{Introduction}

Surface electrochemical processes at real solid electrodes, which are often found in electrocatalytic reactions of interest for energy conversion as well as for the production of substances, involve the participation of adsorption and electroadsorption of species on rough topographies. This is a complex issue of considerable importance for heterogeneous processes at solid/fluid interfaces, to predict the real surface area of a real solid electrode, and to explore the influence of the electrode surface roughness at different scales on the efficiency of those electrode processes. After the advent of fractal geometry, the surface topography covers the range of weakly disordered surfaces that appear at single crystal

\footnotetext{
* Corresponding author. Fax: + 54-221-425-4642.

E-mail address: ajarvia@inifta.unlp.edu.ar (A.J. Arvia).

${ }^{1}$ Visiting Professor at the University of La Plata (Chemistry Department) from Departamento de Química-Física Aplicada, Facultad de Ciencias, Universidad Autónoma, Madrid, Spain.
}

domains, i.e. from solid surfaces with intrinsic (corners, steps, kinks, etc.) or with extrinsic surface defects (inclusions, adsorbed impurities, etc.), to strongly disordered surfaces usually found in polycrystalline branched, dendritic, columnar, etc., materials [1].

Since a few decades ago, for technical reasons in electrochemistry, among rough surfaces special attention has been paid to the structure of porous electrodes, and a number of models with different pore geometries [2-6] have been proposed to account for the behaviour of those electrodes. Otherwise, among rough surface electrodes, columnar structured polycrystalline metallic electrodes constitute promising models to study the influence of surface roughness on relatively simple surface electrochemical processes at solid electrodes [7,8]. However, despite the relevance of these studies for applied electrochemistry, modelling of simple processes such as molecular adsorption on rough surfaces has been mostly restricted to non-electrochemical systems [9-11]. Therefore, efforts to contribute to this issue for electrochemical systems are particularly attractive. 
For molecular adsorption on rough surfaces the role of adsorbate-substrate interactions in affecting the interpretation of adsorption isotherms has long been recognised [9]. Thus, steric effects for surface domains with an average size close to the adsorbate cross section usually appear as an excluded area or excluded volume for adsorption. These effects affect the evaluation of the real surface area of a solid by physical adsorption $[10,11]$ making the interpretation of physico-chemical data derived from adsorption isotherms rather uncertain [9-14].

The fundamental interpretation of excluded area effects turns out to be more difficult when adsorbate-adsorbate interactions and energy barriers for surface diffusion at different crystallographic defects of the irregular substrate are considered. For such a complex situation, Monte Carlo simulations provide, in this case, a possible way to discriminate among those various effects that perturb adsorption measurements [1517].

Recently, Monte Carlo simulations for molecular adsorption on a columnar structured solid substrate 2D profile $[17,18]$ based on a simple Langmuirian model have been performed to determine how the diameter and depth of pores influence data derived from the analysis of adsorption isotherms. In this case, the behaviour of molecular adsorption on a columnar structured substrate has been compared to that occurring on an ideally smooth substrate. Results from the model indicate a marked influence of the width and length of $\mathrm{nm}$-sized pores at the substrate on data derived from isotherms. Thus, the estimated Gibbs free energy of adsorption decreases as the pore width is decreased and the pore depth is increased, i.e. it decreases with the roughness of the substrate $(R)$ defined as the length ratio between the columnar structured and the smooth surface profile. In the context of these simulations $R$ was taken as a coefficient accounting for the increase in real surface area when one moves from the ideal smooth to the columnar structured surface. Data analysis also showed the possibility of cancelling the effect of surface roughness on the adsorption parameters by introducing a correction factor $(f)$ that depends on $R$ according to the relationship $f \propto R^{-n}$, the exponent $n$ changing from $1 / 5$ to 1 as the pore diameter approaches the adsorbate cross section. This model was used to interpret the anomalous behaviour of adsorption data of pyridine from acid solution on columnar structured gold [18].

This paper describes an extension of the preceding molecular adsorption model on a columnar structured topography in which repulsive adsorbate-adsorbate interactions are considered. Monte Carlo simulations allowed us to accomplish a reasonable understanding of the influence of the substrate pore depth and width on the adsorption isotherm when repulsive adsorbate-ad- sorbate interactions are involved. The adsorption isotherms derived from the model are compared to those that have been determined from the adsorption of 1,10-phenantroline (oph) in solution on columnar structured gold electrodes at applied potentials $(E)$ more positive than the potential of zero charge of gold $\left(E_{\mathrm{pzc}}\right)$ and $298 \mathrm{~K}$ [19]. This type of topography was prepared from polycrystalline gold following the procedures indicated elsewhere [20].

\section{The model}

A full description of the model was given in Refs. $[17,18]$. Briefly, a square lattice was used for the 2D Monte Carlo simulations. The system consisted of a cross section of a real three-dimensional (3D) solid (the substrate) in contact with particles confined within a reservoir (the solution).

The solid surface was represented by a continuous 2D profile involving pore width $d_{\mathrm{p}}$, depth $h_{\mathrm{p}}$, and top column width $d_{\mathrm{c}}$ (Fig. 1). No substrate relaxation induced by adsorbates was considered. Dimensionless geometric parameters were scaled with respect to $d_{\mathrm{m}}$, the average diameter of the adsorbate. Thus, $h_{\mathrm{p}}=$ pore

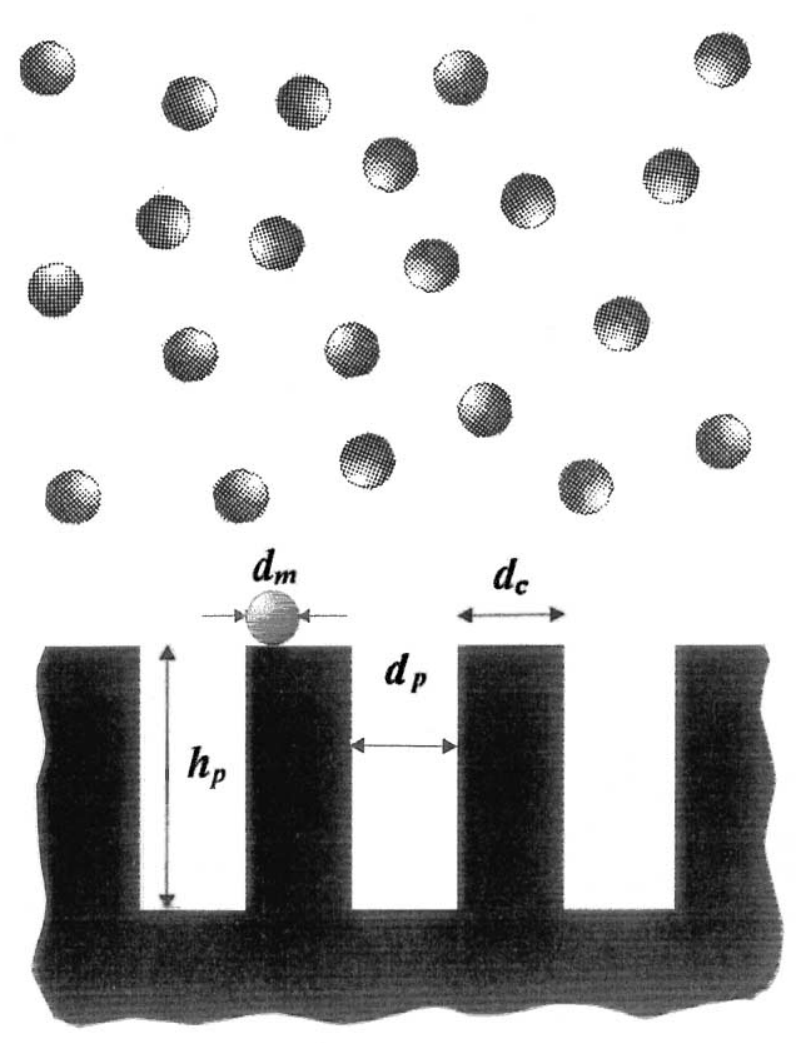

Fig. 1. 2D profile of the columnar-structured substrate in contact with adsorbable particles in the reservoir. The diameter $\left(d_{\mathrm{m}}\right)$ of the adsorbable particles is used as a yardstick. Geometric characteristics of the substrate are $h_{\mathrm{p}}=5 d_{\mathrm{m}}$ and $d_{\mathrm{p}}=2 d_{\mathrm{m}}$. 


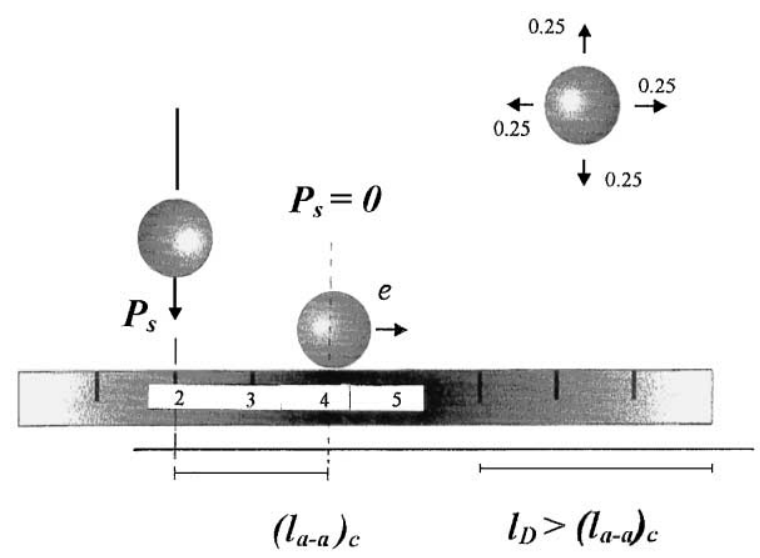

Fig. 2. 2D profile of a smooth substrate. The profile is given on the $d_{\mathrm{m}}$ scale. The displacement probability of particles in the reservoir is 0.25 in each one of the four directions. $P_{\mathrm{s}}$ represents the sticking probability of a particle from the reservoir on the substrate. The attachment of a particle at site 2 implies the displacement of the adsorbed particle at site 4 to a distance $l_{\mathrm{D}}>\left(l_{\mathrm{a}-\mathrm{a}}\right)_{\mathrm{c}}$, where $l_{\mathrm{D}}$ is the surface diffusion length imposed on the model. In this case, $l_{\mathrm{D}}=3 d_{\mathrm{m}}$, and $\left(l_{\mathrm{a}-\mathrm{a}}\right)_{\mathrm{c}}=2 d_{\mathrm{m}}$. The value of $P_{\mathrm{s}}$ changes along $\left(l_{\mathrm{a}-\mathrm{a}}\right)_{\mathrm{c}}$ from $P_{\mathrm{s}}$ at site 2 to $P_{\mathrm{s}}=0$ at site 4 .

$\operatorname{depth} / d_{\mathrm{m}} ; \quad d_{\mathrm{c}}=$ column top diameter $/ d_{\mathrm{m}} ; d_{\mathrm{p}}=$ pore width $/ d_{\mathrm{m}} ; \quad l_{\mathrm{a}-\mathrm{a}}=$ distance between neighbour adsorbates $/ d_{\mathrm{m}}$ (Fig. 2). Values of $d_{\mathrm{p}}, d_{\mathrm{c}}$, and $h_{\mathrm{p}}$ were changed in the ranges $2 \leq d_{\mathrm{p}} \leq n ; 2 \leq d_{\mathrm{c}} \leq n ; 1 \leq h_{\mathrm{p}} \leq n^{\prime} ; n \leq 8$, and $n^{\prime} \leq 60$ are integers. Considering that all surface sites are potentially useful for adsorption, irrespective of the topography, the roughness factor of the substrate was then defined as $R=S / S_{\mathrm{o}}$, in which $S$ and $S_{\mathrm{o}}$ are the number of adsorption sites of length $d_{\mathrm{m}}$ at the columnar structured and smooth surface substrate, respectively.

Effective repulsive adsorbate-adsorbate interactions appear for $l_{\mathrm{a}-\mathrm{a}} \leq\left(l_{\mathrm{a}-\mathrm{a}}\right)_{\mathrm{c}}$, where $\left(l_{\mathrm{a}-\mathrm{a}}\right)_{\mathrm{c}}$, denotes the critical distance beyond which those repulsive interactions no longer exist. The value of $l_{\mathrm{D}}$ is the maximum surface diffusion length imposed on the adsorbate.

Particles representing adsorbable molecules in the solution were randomly distributed in the reservoir (Fig. 2). In this simulation procedure a spatial random walk of particles is involved. No particle-particle interaction in the solution was taken into account. The movement of particles was simulated as follows. Both a particle and one of its neighbour sites were selected at random with the same probability in the square lattice. The selected particle was allowed to jump to an unoccupied neighbour site. Otherwise, when the neighbour site is occupied the change in the location of the particle is forbidden. The Monte Carlo time unit $\left(t_{\mathrm{MC}}\right)$ was defined as the time required to allow all particles to move once on average. Therefore, the adsorption time $\left(t_{\mathrm{ad}}\right)$ was defined as the product $N t_{\mathrm{MC}}, N$ being the number of Monte Carlo units.
Particles contacting the surface (Fig. 2) were adsorbed with a probability $P_{\mathrm{s}}$ and desorbed with a probability $P_{\mathrm{d}}=1-P_{\mathrm{s}}$. Typical values of $P_{\mathrm{s}}$ were $0.999,0.98,0.95$ and 0.93 . It should be noted that for a constant concentration of particles in the environment (c) in real electrochemical systems at constant temperature, values of $P_{\mathrm{d}}$ and $P_{\mathrm{s}}$ depend on $c$ and, depending on the kinetic control of the adsorption reaction, on $E$, the electric potential at the substrate/environment interface. When $l_{\mathrm{a}-\mathrm{a}} \leq\left(l_{\mathrm{a}-\mathrm{a}}\right)_{\mathrm{c}}$, for all surface directions, the value of $P_{\mathrm{s}}$ of incoming particles is decreased linearly from one of the above mentioned values of $P_{\mathrm{s}}$ for $l_{\mathrm{a}-\mathrm{a}}=\left(l_{\mathrm{a}-\mathrm{a}}\right)_{\mathrm{c}}+1$ to $P_{\mathrm{s}}=0.05$ at the nearest site where the initial adsorbate was present. This fact implies that $P_{\mathrm{s}}$ decreases as the degree of surface coverage by adsorbates $(\theta)$ increases. In the range $l_{\mathrm{D}}>\left(l_{\mathrm{a}-\mathrm{a}}\right)_{\mathrm{c}}$, the surface diffusion of adsorbates is free of repulsive force constraints. Conversely, when the incoming particle is adsorbed within the domain $l_{\mathrm{a}-\mathrm{a}} \leq\left(l_{\mathrm{a}-\mathrm{a}}\right)_{\mathrm{c}}$, it is allowed to diffuse on the substrate surface either to satisfy the condition $l_{\mathrm{a}-\mathrm{a}} \leq\left(l_{\mathrm{a}-\mathrm{a}}\right)_{\mathrm{c}}+1$ or, if it is trapped between two adsorbed particles, to shift to a site in which the lowest adsorbate-adsorbate repulsive interaction is involved.

The value of $\theta$ was evaluated from the snapshots as the ratio between the number of adsorbed particles and the number of adsorption sites, $S$. Therefore, the definition of $\theta$ involves the "real surface area" of the system, i.e. the entire pore area (wall and bottom) as well as the area in between pores. In real electrochemical systems this is equivalent to measuring $R$ using as a yardstick an atom or a molecule of size smaller than the smallest pore cross section. The simulation routine was continued until an apparent constant surface coverage $\left(\theta_{\mathrm{e}}\right)$ was attained. For practical reasons, the value of $\theta_{\mathrm{e}}$ was chosen when $\Delta \theta / \Delta t_{\mathrm{ad}}<10^{-5} \mathrm{~s}^{-1}$, i.e. the unidirectional rate of adsorption approaches the unidirectional rate of desorption. These values of $\theta_{\mathrm{e}}$ were taken as if they were the equilibrium values. Periodic boundary conditions were used to eliminate size effects and to maintain constant the number of particles in the reservoir as the adsorption process took place.

\section{Monte Carlo simulations}

\subsection{Parameters used in the simulations}

For a constant $P_{\mathrm{s}}$, two limiting initial adsorption situations can be simulated. The first one started when the solid surface contacts the environment having pores empty of adsorbable particles because of their very slow transport into pores. Then, the initial concentration of adsorbable particles in the pores is $c_{\mathrm{p}}=0$. Otherwise, for the second limiting situation, the initial concentration of adsorbable particles in the pores $\left(c_{\mathrm{p}}\right)$ and at the 
environment $(c)$ are the same. This situation would correspond to a rapid diffusion of particles from the environment into pores to attain equilibrium before the simulation was started. This second alternative, however, is beyond the scope of the present work.

For the first limiting situation, typical snapshots resulting from profiles with different values of $d_{\mathrm{m}}, d_{\mathrm{p}}, P_{\mathrm{s}}$ and $t_{\text {ad }}$ were obtained (Fig. 3). As $t_{\text {ad }}$ is increased, the value of $\theta$ gradually increases, and then two types of adsorbates can be distinguished, namely, adsorbates on column tops and on pore walls. A detailed inspection of the snapshots reveals that, occasionally, neighbour particles adsorbed at opposite wall sites produce a plugged bottleneck effect and pore tortuosity. When $d_{\mathrm{p}}$ is increased, these effects tend to diminish.

\subsection{Adsorption isotherms}

To determine the adsorption isotherm, values of $\theta_{\mathrm{e}}$ derived from Monte Carlo simulations for different $c$ and $h_{\mathrm{p}}$ were used. For a constant $h_{\mathrm{p}}$, the value of $\theta_{\mathrm{e}}$ increases with $c$ (Fig. 4a), but for a constant $c$, the value of $\theta_{\mathrm{e}}$ decreases with $h_{\mathrm{p}}$ due to the excluded volume resulting from the plugged bottleneck effect and pore tortuosity caused by the presence of adsorbates on the pore wall. Data were analysed considering an adsorption isotherm that has been described recently, which includes a $\theta$-dependent Gibbs free energy term [21]. In the absence of applied electric potential, this adsorption isotherm can be written

$\theta_{\mathrm{e}} /\left(1-\theta_{\mathrm{e}}\right)=x \exp \left[-\Delta G^{\circ}\left(\theta_{\mathrm{e}}\right) / R T\right]$

where $x$ is the molar fraction of adsorbable species in the solution $\left(x=c /\left(c_{\mathrm{s}}+c\right) \cong c / c_{\mathrm{s}}\right), c_{\mathrm{s}}$ is the molar concentration of the solvent, $T$ is the absolute temperature, $R$ is the universal gas constant, and $\Delta G^{\circ}\left(\theta_{\mathrm{e}}\right)$ is the $\theta_{\mathrm{e}}$-dependent standard Gibbs free energy of $2 \mathrm{D}$ adsorption [21].

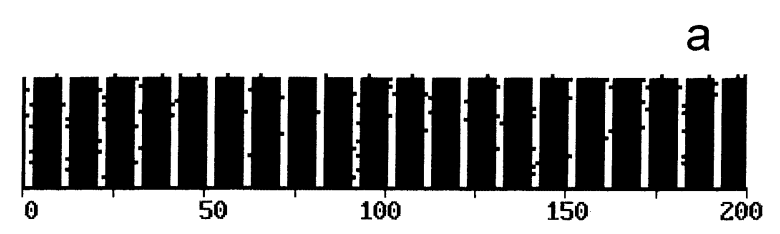

b

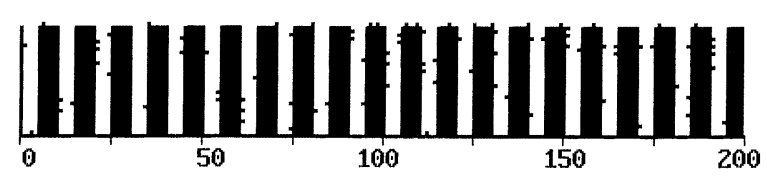

Fig. 3. Monte Carlo snapshots. (a) $d_{\mathrm{p}}=2 ; d_{\mathrm{c}}=8 ; h_{\mathrm{p}}=30 ; c=2.76 \times$ $10^{13}$ particles $\mathrm{cm}^{-2} ; P_{\mathrm{s}}=0.95 ;\left(l_{\mathrm{a}-\mathrm{a}}\right)_{\mathrm{c}}=2 ; t_{\mathrm{ad}}=439 ; \theta_{\mathrm{e}}=0.063$. (b) $d_{\mathrm{p}}=4 ; d_{\mathrm{c}}=6 ; h_{\mathrm{p}}=30 ; c=2.76 \times 10^{13} ; P_{\mathrm{s}}=0.95 ; t_{\mathrm{ad}}=35 ;\left(l_{\mathrm{a}-\mathrm{a}}\right)_{\mathrm{c}}=2 ;$ $\theta_{\mathrm{e}}=0.062$. Pore entrance blocking and plugged bottleneck effect at some pores can be distinguished.
$\Delta G^{\circ}\left(\theta_{\mathrm{e}}\right)=\Delta G^{\circ}\left(\theta_{\mathrm{e}}=0\right)+g\left(\theta_{\mathrm{e}}\right)$

In Eq. (2), $\Delta G^{\circ}\left(\theta_{\mathrm{e}}=0\right)$ is the standard Gibbs free energy of adsorption for null coverage, $g\left(\theta_{\mathrm{e}}\right)$ is a $\theta_{\mathrm{e}}$-dependent parameter which describes adsorbate-adsorbate interactions.

Considering the type of adsorbate-adsorbate interactions assumed in the model, data from Monte Carlo simulation plotted as $\ln \left[\theta_{\mathrm{e}} /\left(1-\theta_{\mathrm{e}}\right) c\right]$ versus $\theta_{\mathrm{e}}$ plots yielded a family of straight lines with different slope and ordinate for $\theta_{\mathrm{e}}=0$ (Fig. 4b). This kind of linear plots are predicted by Eqs. (1) and (2) provided that $\Delta G^{\circ}\left(\theta_{\mathrm{e}}\right)$ changes linearly with $\theta_{\mathrm{e}}$. Accordingly, Eq. (1) can be reformulated as the empirical Frumkin adsorption isotherm [22,23].

$$
\begin{aligned}
\theta_{\mathrm{e}} /\left(1-\theta_{\mathrm{e}}\right) & =x \exp \left[-\Delta G^{\circ}\left(\theta_{\mathrm{e}}\right) / R T\right] \\
& =x \exp \left[-\Delta G^{\circ}\left(\theta_{\mathrm{e}}=0\right)\right] \exp \left[-2 a \theta_{\mathrm{e}}\right]
\end{aligned}
$$

where the parameter $a$ is a measure of adsorbate-adsorbate interactions. Negative values of $a$ indicate the prevalence of lateral repulsive interactions.

Values of $a$ and $\Delta G^{\circ}\left(\theta_{\mathrm{e}}=0\right)$ were estimated from the slope and ordinate of Frumkin isotherm plots (Fig. 4b), respectively, considering that $c_{\mathrm{s}}=55.5 \mathrm{M}$ as it would be the case of adsorbable species in aqueous solution. The value of $a$ increases negatively with $R$ (Fig. 5a). For constant $R$, and in the range $2 \leq l_{\mathrm{a}-\mathrm{a}} \leq 4$, the value of $a$ turns out to be more negative as $l_{\mathrm{a}-\mathrm{a}}$ is increased (Fig. 5a). Otherwise, values of $\Delta G^{\circ}\left(\theta_{\mathrm{e}}=0\right)$ become less negative as $R$ increases, irrespective of $l_{\mathrm{a}-\mathrm{a}}$ (Fig. 5b). In the range of $R$ covered by our simulations the value of $\Delta G^{\circ}\left(\theta_{\mathrm{e}}=0\right)$ changes from $-15 \pm 2$ to $-10 \pm 2 \mathrm{~kJ}$ $\mathrm{mol}^{-1}$. For all values of $R, \Delta G^{\circ}\left(\theta_{\mathrm{e}}=0\right)$ increases negatively with $\ln P_{\mathrm{s}} /\left(1-P_{\mathrm{s}}\right.$ ) (Fig. 5c), and for $P_{\mathrm{s}}<0.99$, data approach linear relationships with a slope close to $-R T / 2$. For this case, the relationship between $P_{\mathrm{s}}$ and $\Delta G^{\circ}\left(\theta_{\mathrm{e}}=0\right)$ can be expressed as

$\ln P_{\mathrm{s}} /\left(1-P_{\mathrm{s}}\right) \approx-2 \Delta G^{\circ}\left(\theta_{\mathrm{e}}=0\right) / R T$

Therefore, the dependences on $R$ of both $a$ and $\Delta G^{\circ}\left(\theta_{\mathrm{e}}=0\right)$ are mainly due to pure geometric factors related to excluded pore area effects. Then, the influence of geometric factors on the adsorption parameters can be cancelled introducing a $R$-dependent empirical correction factor $(f)$ in Eq. (1), defined as the ratio

$f=\left(\theta_{\mathrm{e}}\right)_{\mathrm{ss}} /\left(\theta_{\mathrm{e}}\right)_{\mathrm{cs}}=K^{\prime} R$

where $\left(\theta_{\mathrm{e}}\right)_{\mathrm{ss}}$ and $\left(\theta_{\mathrm{e}}\right)_{\mathrm{cs}}$ are the surface coverage by adsorbates for the smooth surface and the columnar structured substrate, respectively, and $K^{\prime}$ is a geometric factor that depends on the set of parameters used in the simulations, being $K^{\prime} \geq 1$. For the smooth substrate $K^{\prime}=1$ so that for $R \rightarrow 1, f \rightarrow 1$, i.e. in this case the substrate surface is fully accessible for particle adsorption. Therefore, the $f$ versus $R$ plots approach straight lines going through $R=1$ (Fig. 6). Therefore, combining equation Eqs. (3) and (4). 

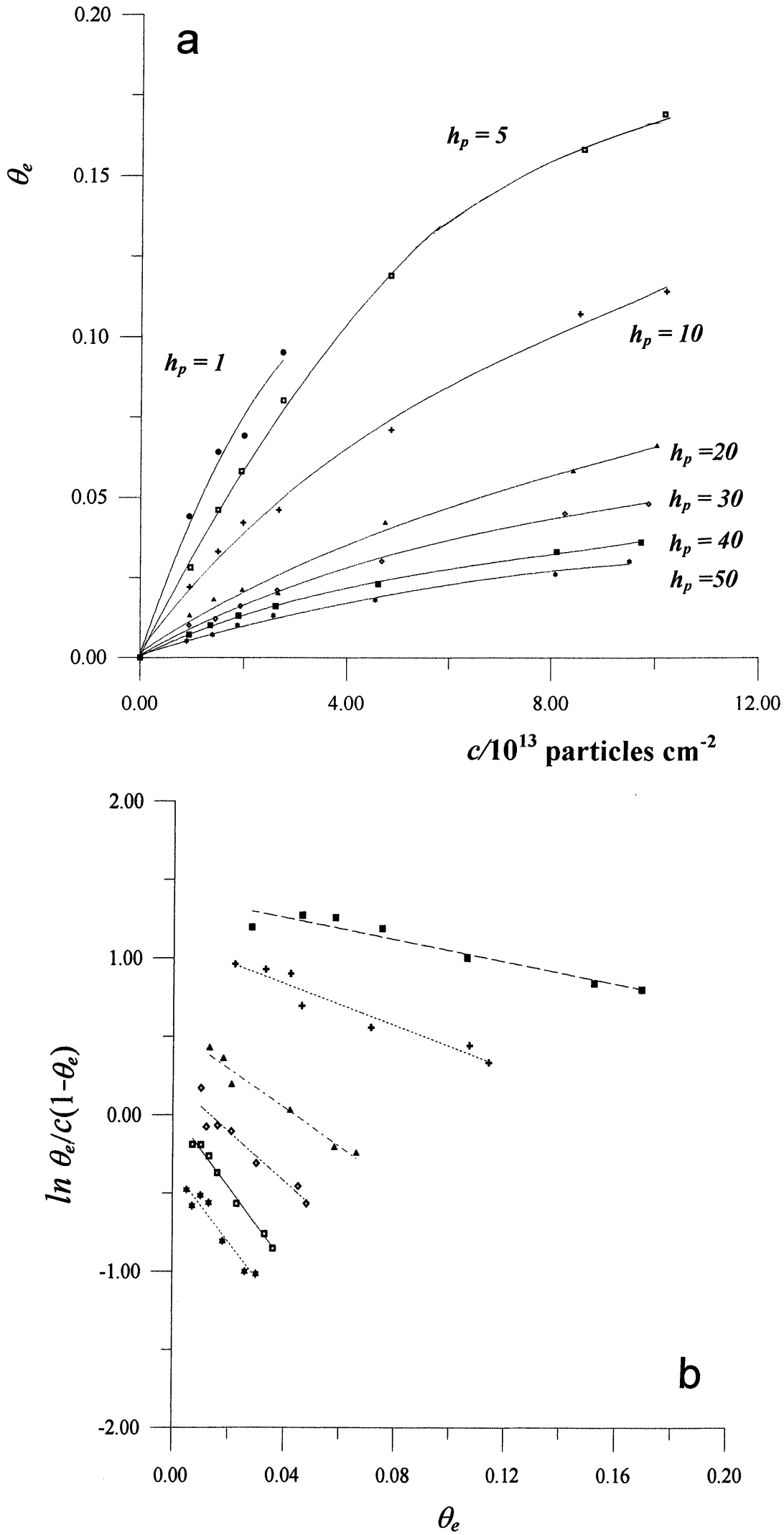

Fig. 4. (a) Plots of $\theta_{\mathrm{e}}$ vs. c. $P_{\mathrm{s}}=0.95 ; d_{\mathrm{p}}=2 d_{\mathrm{m}} ;\left(l_{\mathrm{a}-\mathrm{a}}\right)_{\mathrm{c}}=2 d_{\mathrm{m}}$; and different values of $h_{\mathrm{p}}$. (b) Plots of adsorption data according to Frumkin isotherm. Data taken from Fig. 4a. 

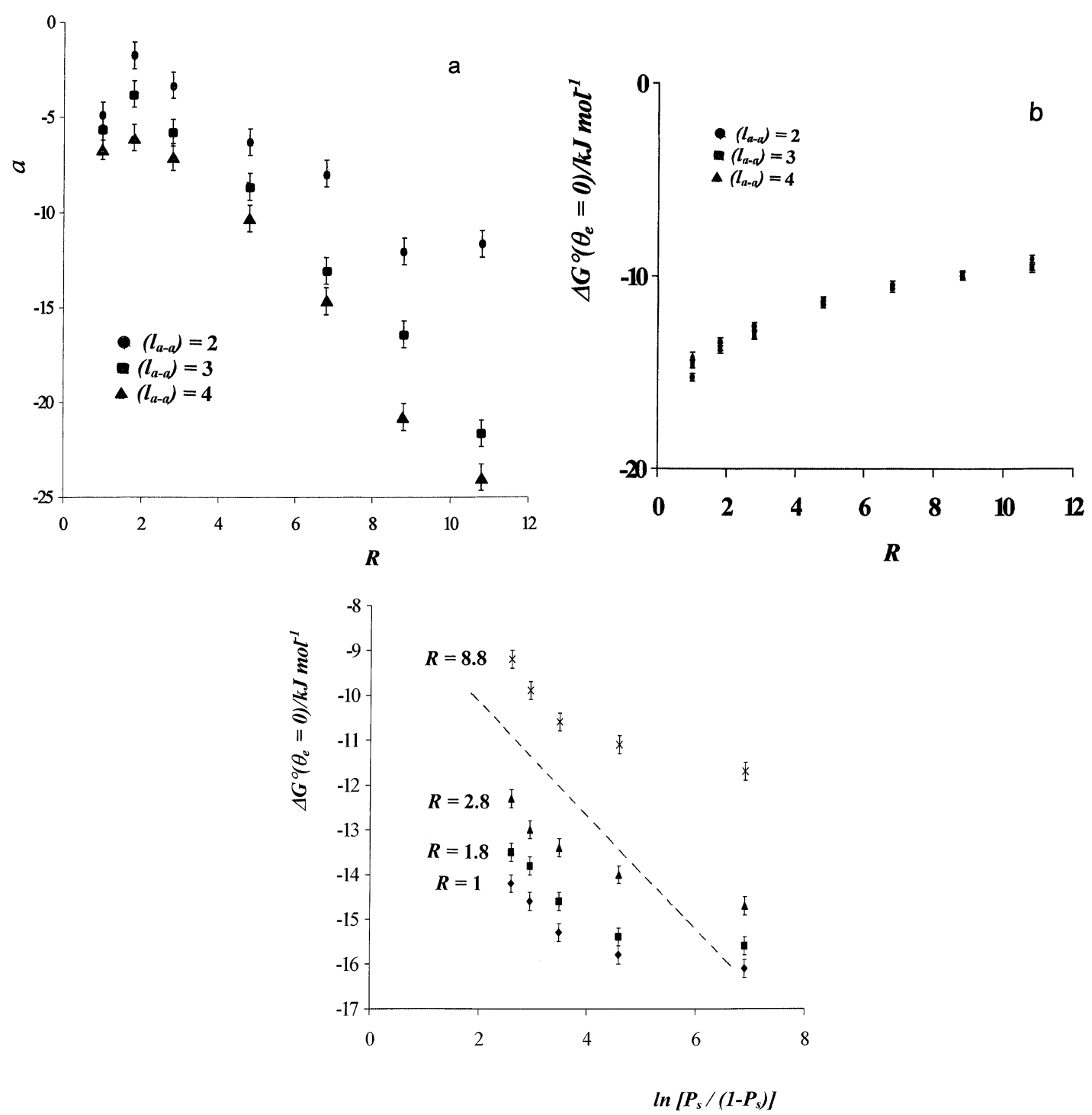

Fig. 5. (a) Dependence of the value of $a$ on $R$ obtained from $2 \mathrm{D}$ Monte Carlo simulations. $P_{\mathrm{s}}=0.95 ; d_{\mathrm{p}}=2 d_{\mathrm{m}}$ and different values of $\left(l_{\mathrm{a}-\mathrm{a}}\right)_{\mathrm{c}}$. (b) Dependence of $\Delta G^{\circ}\left(\theta_{\mathrm{e}}=0\right)$ on $R$ resulting from 2D Monte Carlo simulations. $P_{\mathrm{s}}=0.95 ; d_{\mathrm{p}}=2 d_{\mathrm{m}}$ and different values of $\left(l_{\mathrm{a}-\mathrm{a}}\right)_{\mathrm{c}}$. (c) Plot of $\Delta G^{\circ}\left(\theta_{\mathrm{e}}=0\right)$ vs. $\ln \left[P_{\mathrm{s}} /\left(1-P_{\mathrm{s}}\right)\right]$ for different values of $R$. The slope of the dashed trace corresponds to $R T / 2$.

$f \theta_{\mathrm{e}} /\left(1-f \theta_{\mathrm{e}}\right)=x \exp \left[-\Delta G^{\circ}\left(\theta_{\mathrm{e}}=0\right) / R T\right] \exp \left[-2 a f \theta_{\mathrm{e}}\right]$

Eq. (6) leads to a single isotherm in the $f \theta_{\mathrm{e}}$ versus $c$ plot (Fig. 7a), with $\Delta G^{\circ}\left(\theta_{\mathrm{e}}=0\right)=-12 \pm 2 \mathrm{~kJ} \mathrm{~mol}^{-1}$, irrespective of $R$.

For a Frumkin-type electrochemical adsorption processes [21-23], Eq. (6) results in

$$
\begin{aligned}
& f \theta_{\mathrm{e}} /\left(1-f \theta_{\mathrm{e}}\right) \\
& =x \exp [-z F E / R T] \exp \left[-\Delta G^{\circ}\left(\theta_{\mathrm{e}}=0\right) / R T\right] \\
& \quad \exp \left[-2 a f \theta_{\mathrm{e}}\right]
\end{aligned}
$$

where $z$ is the number of electrons involved in each adsorption event and $E$ is the electric potential applied to the electrode/solution interface. For adsorption under constant potential, $\exp [-z F E / R T]=K^{\prime \prime}$, and Eq. (7) can be written

$$
\begin{aligned}
& f \theta_{\mathrm{e}} /\left(1-f \theta_{\mathrm{e}}\right) \\
& =x K^{\prime \prime} \exp \left[-\Delta G^{\circ}\left(\theta_{\mathrm{e}}=0\right) / R T\right] \exp \left[-2 a f \theta_{\mathrm{e}}\right]
\end{aligned}
$$

Then, the $K^{\prime \prime}$ can be used as a scale adjusting parameters to compare model and experimental data.

\subsection{Testing the adsorption model}

Results from the 2D model can be qualitatively compared to those obtained for the adsorption of oph on columnar structured gold substrates for $E>E_{\mathrm{pzc}}$ (gold) $=-0.45 \mathrm{~V}$ from aqueous $5 \times 10^{-4} \mathrm{M} \leq c_{\mathrm{oph}} \leq$ $5 \times 10^{-3} \mathrm{M}+0.1 \mathrm{M}$ perchloric acid at $298 \mathrm{~K}$ using chronocoulometry [19]. The surface structure of this type of substrate has been characterised by scanning tunnelling microscopy. Its topography can be described as an array of nm-sized columns separated by pores of 


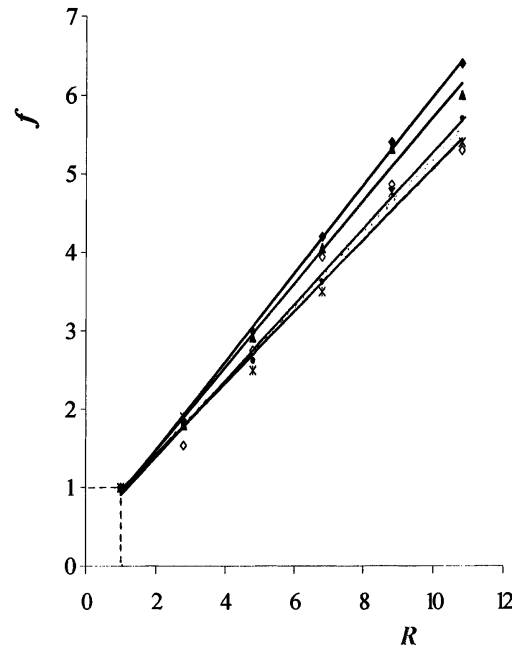

Fig. 6. Plots of $f$ vs. $R$ for different sets of simulation parameters: $P_{\mathrm{s}}=0.999,\left(l_{\mathrm{a}-\mathrm{a}}\right)_{\mathrm{c}}=3, d_{\mathrm{p}}=2 ;(\boldsymbol{\Delta}) P_{\mathrm{s}}=0.99,\left(l_{\mathrm{a}-\mathrm{a}}\right)_{\mathrm{c}}=3, d_{\mathrm{p}}=2 ;(\diamond)$ $P=0.97,\left(l_{\mathrm{a}-\mathrm{a}}\right)_{\mathrm{c}}=3, d_{\mathrm{p}}=2 ;(\circlearrowleft) P_{\mathrm{s}}=0.95,\left(l_{\mathrm{a}-\mathrm{a}}\right)_{\mathrm{c}}=2, d_{\mathrm{p}}=6 ;(*)$ $P_{\mathrm{s}}=0.93,\left(l_{\mathrm{a}-\mathrm{a}}\right)_{\mathrm{c}}=3, d_{\mathrm{p}}=2$.

$\mathrm{nm}$ range diameter and depths in the $\mathrm{nm}-\mu \mathrm{m}$ range. The size of the smallest pores are in the 1-2 $\mathrm{nm}$ range [18]. The value of $R$ for columnar structured gold electrodes was determined from the ratio of the oxygen adatom monolayer electrodesorption charge at these electrodes to that at the smooth electrode. When both
$R$ and $c_{\text {oph }}$ were kept constant, the value of $\theta_{\mathrm{e}}$ diminished as $R$ was increased. For very rough substrates such as columnar structured gold surfaces, for the time scale used in the electroadsorption measurements values of $\theta_{\mathrm{e}}$ can be considered as real equilibrium values.

The adsorption of oph on columnar structured gold substrates with roughness in the range $1<R<50$ fulfils isotherms in which adsorbate-adsorbate repulsive interactions play a relevant role [19]. It should be noted that a better comparison between simulation and experimental data can be made by normalising the concentration scale in Fig. 7a,b in terms of $x$. For this purpose, in the model we assumed that the profile of the $2 \mathrm{D}$ substrate is the same in the $x$-and $y$-coordinates, the average volume concentration of adsorbable particles in the environment is constant, and voids in the environment are statistically occupied by water molecules $\left(c_{\mathrm{s}}=55.5 \mathrm{M}\right)$. In agreement with the model (Fig. 7a), data from the experimental system (Fig. 7b) overlap on a single $f \theta_{\mathrm{e}}$ versus $c_{\text {oph }}$ plot, irrespective of $R$.

The dependence of $a$ on $R$ from the model compares qualitatively well with that observed for the experimental system (Fig. 8a). Thus, both model and experimental data exhibit a decrease in the value of $a$ with $R$, as expected for an apparent increase in the lateral repulsive interactions with $R$. Conversely, the comparison of
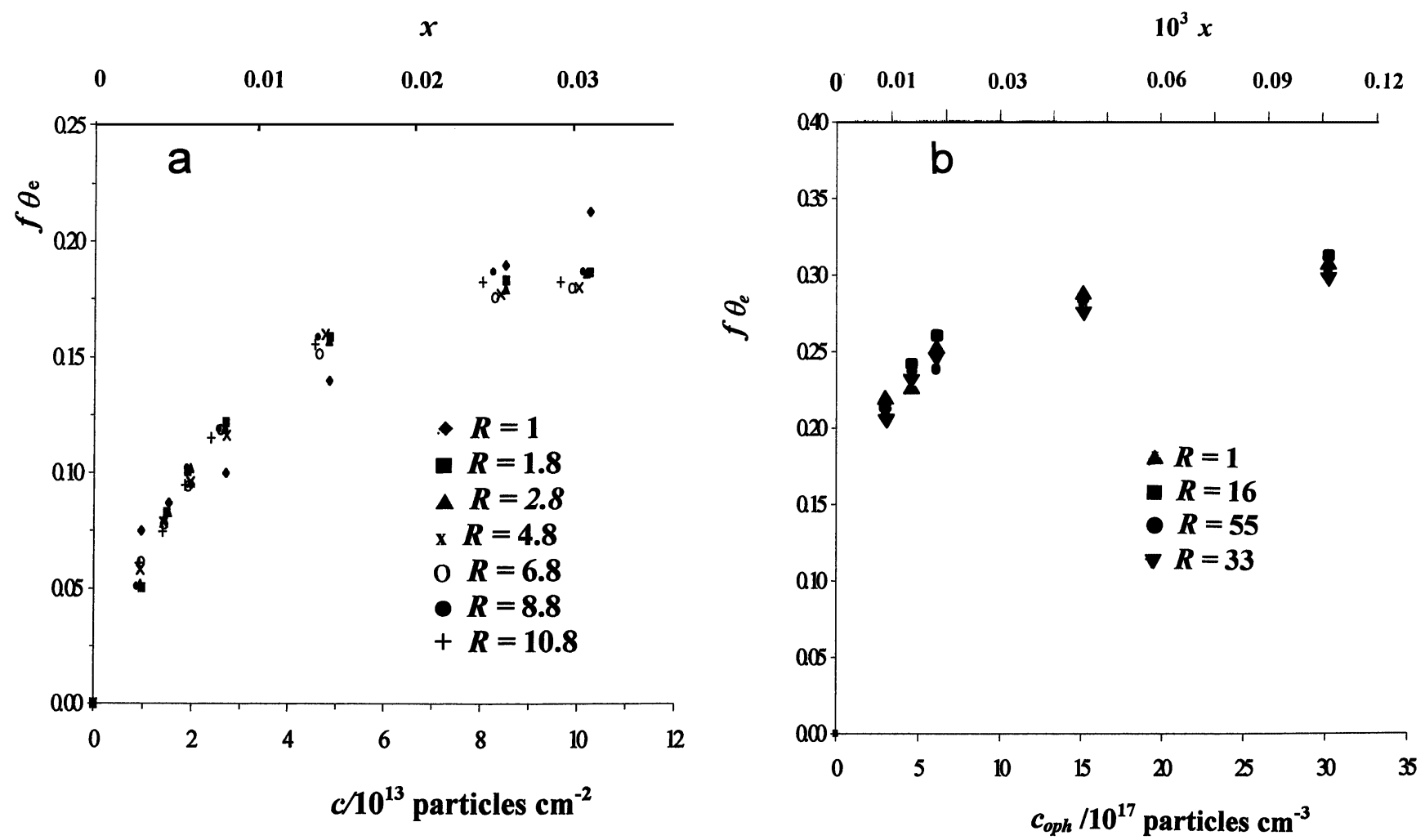

Fig. 7. Plots of $f \theta_{\mathrm{e}}$ vs. $c$. (a) 2D Monte Carlo simulations $(c=c)$. (b) Adsorption of oph from aqueous solution on columnar structured gold electrodes $\left(c=c_{\text {oph }}\right)$. 

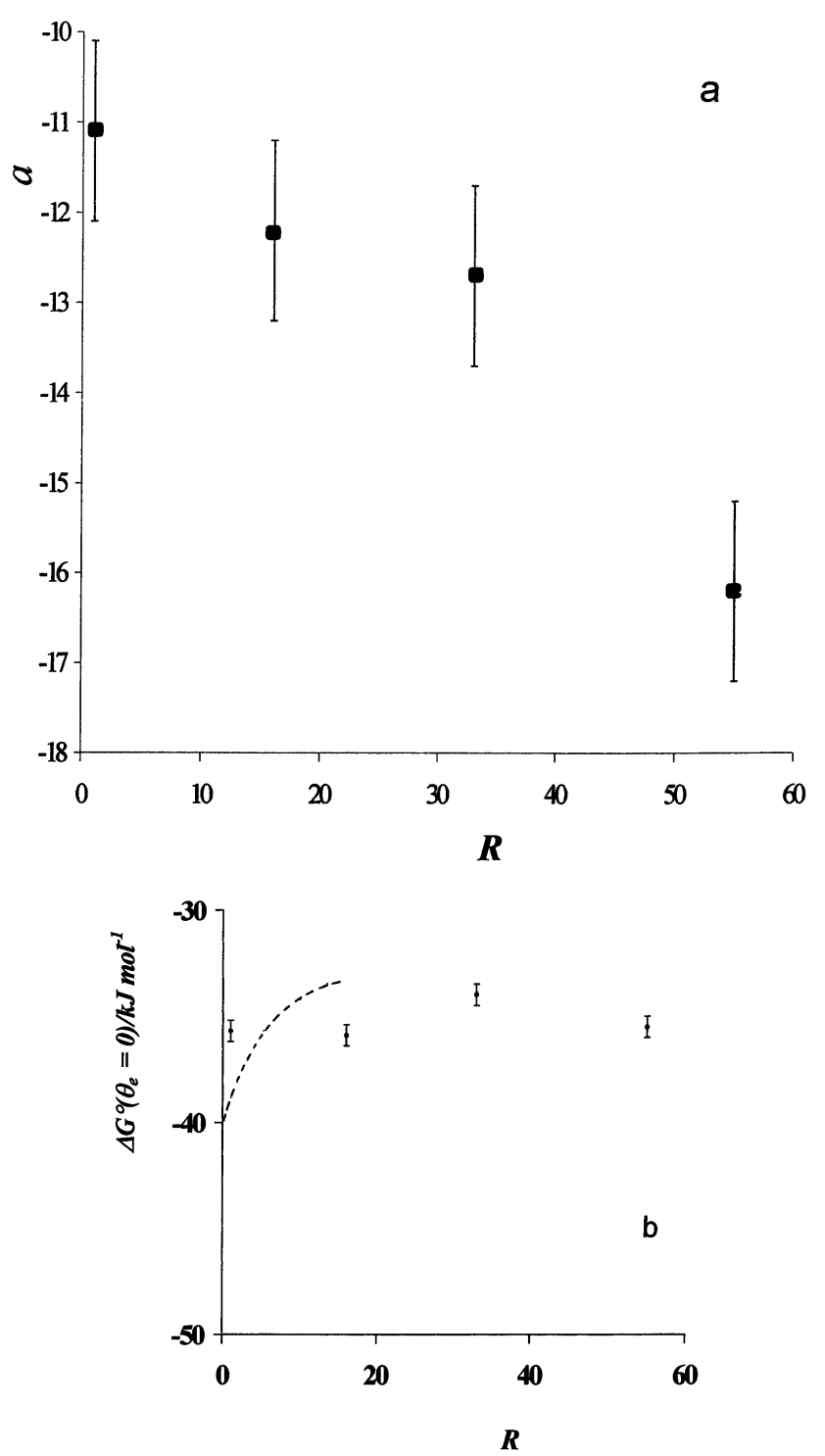

Fig. 8. Dependences of $a$ on $R$ (a) and $\Delta G^{\circ}\left(\theta_{\mathrm{e}}=0\right)$ on $R$ (b). Adsorption of oph from aqueous solution on columnar structured gold electrodes at $298 \mathrm{~K}$. To facilitate data comparison in Fig. 8b, the $\Delta G^{\circ}\left(\theta_{\mathrm{e}}=0\right)$ vs. $R$ plot from the model (dashed trace), after an arbitrary shift of values of $\Delta G^{\circ}\left(\theta_{\mathrm{e}}=0\right)$ on the ordinate, is included.

the dependence of model (Fig. 5a) and experimental (Fig. 8b) values of $\Delta G^{\circ}\left(\theta_{\mathrm{e}}=0\right)$ on $R$, after an arbitrary shift of model data, shows some differences. In fact, for Langmuirian adsorption on a particular polycrystalline substrate involving crystallographic features which are substantially independent of roughness, one might consider an almost constant experimental value of $\Delta G^{\circ}\left(\theta_{\mathrm{e}}=0\right)$ emerging from a compensation that would result if the adsorption enthalpy contribution decreases and the entropy contribution becomes less negative as $R$, i.e. $\theta_{\mathrm{e}}$, increases [21,24]. However, values of $\Delta G^{\circ}\left(\theta_{\mathrm{e}}=0\right)$ from the model depend on $R$.

There are various possible explanations for the difference depicted in Fig. 5a and Fig. 8b. First, it should be noted that for extremely long $t_{\mathrm{ad}}$, the value of $\theta$ derived from the model increases continuously although very slowly. This would mean that true adsorption equilibrium has not been attained, at least in the time scale of our simulations. In fact, several features contribute to slow down the rate of adsorption at pores, namely, a hindrance of surface diffusion of particles at pore wall due to the presence of adsorbates at the upper level of the wall, and changes in tortuosity along the pore. Accordingly, the model reveals that for real rough systems it would be difficult to establish whether true thermodynamic equilibrium was attained, probably because of the hysteresis of the adsorption/desorption processes taking place at nm-size pores [12]. Secondly, the large gap between the model and the real testing system has to be considered as well. In fact, in our simulations the pore diameter is always greater than the diameter of the adsorbing particle, a situation that is seldom found in real systems, in which the substrate usually exhibits a broad pore size distribution. Besides, the model does not distinguish between values of $P_{\mathrm{d}}$ from the flat region of the surface and from pore walls. For small diameter pores, the adsorption energy for an adsorbate located at the pore wall should be influenced by the presence of the bare pore wall just in front of it. Furthermore, for electrochemical adsorption experiments on rough conducting surfaces the penetration of the electric signal into the pore region is rather poor and the number of solution constituents makes interfacial phenomena much more complicated [2-8]. All these effects might contribute to facilitate the attainment of equilibrium more easily at the experimental system. Nevertheless, despite these possible explanations it should be noted that the errors involved in both the experimental and modelling data constitute a limiting factor to explain the difference in the dependence of values of $\Delta G^{\circ}\left(\theta_{\mathrm{e}}=0\right)$ on $R$. More accurate and extensive experimental and modelling data would be required for its understanding.

Finally, the correlation between experimental data and modelling including lateral repulsive interactions confirms the importance of the excluded volume effect for the adsorption of molecules on surfaces with nmsized pores, i.e. pores of the order of or slightly larger than the adsorbate cross section, which is principally reflected in the dependence of $a$ on $R$. The model accounts for results obtained over a relatively wide range of experimental conditions, proving the advantages of Monte Carlo simulation for the analysis of molecular adsorption on solids with a rough topography. Furthermore, adsorption data can be adjusted on a common plot irrespective of $R$ thus offering an interesting possibility to obtain reliable thermodynamic parameters for adsorption on those substrates. It should be noted that the normalisation of data using $f$ is to some extent comparable to the use of $V / V_{\mathrm{m}}$ ratio 
in gas adsorption, where $V$ is the adsorbate volume and $V_{\mathrm{m}}$ is the monolayer volume of the adsorbate on the substrate.

\section{Conclusions}

1. 2D Monte Carlo simulation data for molecular adsorption on columnar structured substrates under adsorbate-adsorbate repulsive interactions fulfil the same isotherm for each set of pore width, pore depth and top column dimensions.

2. As the pore depth increases and pore width approaches the adsorbate molecular size, the surface coverage by adsorbed species decreases sharply.

3. The adsorbate-adsorbate repulsive interaction parameters derived from simulated isotherms show a marked dependence on the substrate roughness factor. This fact is attributed mainly to steric effects of pore width and pore depth on molecular adsorption.

4. The influence of roughness on the isotherms is smoothed down by introducing a correction factor, which allowed us to collapse all simulated isotherms into a single isotherm with consistent isosteric parameters.

5. Predictions from the 2D adsorption model were qualitatively compared to earlier experimental data derived for the adsorption of 1,10-phenantroline on columnar structured gold surfaces with roughness factors in the range $1-33$.

6. The correction factor emerging from the substrate topographic characteristics allowed us to estimate reliable thermodynamic data free from artefacts resulting from the geometry of the substrate.

\section{Acknowledgements}

This work was partially supported by the Consejo Nacional de Investigaciones Científicas y Técnicas (CONICET) (PIP 4376/96 and PIP 0897/98) and Agencia Nacional de Promoción Científica y Tecnológica (PICT 99-5030, PICT 06-03251/98) from Argentina and Proyecto $\mathrm{N}^{\circ}$ PB97-0035, DGICYT from Spain.

\section{References}

[1] R.C. Salvarezza, A.J. Arvia, in: B.E. Conway, J.O'M. Bockris, R.E. White (Eds.), Modern Aspects of Electrochemistry, vol. 28, Plenum Press, New York, 1996, pp. 289-373.

[2] (a) R. de Levie, J. Electroanal. Chem. 10 (1965) 113; (b) R. de Levie, Adv. Electrochem. Electrochem. Eng. 6 (1967) 329

(c) R. de Levie, A. Vogt, J. Electroanal. Chem. 281 (1990) 23.

[3] R.D. Armstrong, D. Eyre, W.P. Race, A. Ince, J. Appl. Electrochem. 1 (1971) 179.

[4] K.J. Euler, Electrochim. Acta 17 (1972) 619.

[5] H. Keiser, K.D. Beccu, M.A. Gutjahr, Electrochim. Acta 21 (1976) 539

[6] M. Keddam, H. Takenouti, C.R. Acad. Sci. Paris 302 (1986) 281.

[7] M.M. Gómez, L. Vázquez, R.C. Salvarezza, J.M. Vara, A.J. Arvia, J. Electroanal. Chem. 317 (1991) 125.

[8] S. Real, J.R. Vilche, A.J. Arvia, J. Electronal. Chem. 341 (1992) 329.

[9] M. Jaroniec, R. Madey, Physical Adsorption on Heterogeneous Solids, Elsevier, Amsterdam, 1988.

[10] D. Avnir (Ed.), The Fractal Approach to Heterogeneous Chemistry, Wiley, New York, 1989.

[11] W. Rudzinski, D.H. Everett, Adsorption of Gases on Heterogeneous Surfaces, Academic Press, London, 1998 chapter 46.

[12] A. Seri-Levi, D. Avnir, Langmuir 9 (1993) 3067.

[13] A. Seri-Levi, D. Avnir, in: Proceedings of the IVth International Conference on Fundamentals of Adsorption, 1992, International Adsorption Society, Kyoto, 1993, p. 365.

[14] P. Pfeifer, Y.J. Wu, M.W. Cole, J. Krim, Phys. Rev. Lett. 62 (1997) 1989

[15] L.D. Gelb, K.E. Gubbies, Langmuir 14 (1998) 2097.

[16] V.A. Bakaev, W.A. Steele, Langmuir 13 (1997) 1054.

[17] M.M. Gómez, J.M. Vara, J.C. Hernández, R.C. Salvarezza, A.J. Arvia, Electrochim. Acta 44 (1998) 1255

[18] M.M. Gómez, J.M. Vara, J.C. Hernández, R.C. Salvarezza, A.J. Arvia, J. Electroanal. Chem. 474 (1999) 74 and references cited therein.

[19] M.M. Gómez, M.P. García, J. San Fabián, L. Vázquez, R.C. Salvarezza, A.J. Arvia, Langmuir 12 (1996) 818.

[20] L. Vázquez, A. Bartolomé, A.M. Baró, R.C. Salvarezza, A.J. Arvia, Surf. Sci. 215 (1989) 171 and references cited therein.

[21] G. Jerkiewicz, A. Zolfaghari, J. Phys. Chem. 100 (1996) 8454.

[22] A.N. Frumkin, Z. Physik. Chem. 116 (1925) 466.

[23] A.N. Frumkin, in: P. Delahay (Ed.), Advances of Electrochemistry and Electrochemical Engineering, vol. 3, Interscience Publishers, New York, 1963, pp. 287-391.

[24] W. Breiter, Electrochim. Acta 7 (1963) 709; in: Electrochemical Processes in Fuel Cells, Springer-Verlag, Berlin, Heidelberg, New York, 1969, p. 56. 